\title{
Direct formation of InAs quantum dots grown on InP (001) by solid-source molecular beam epitaxy
}

\author{
David Fuster, Antonio Rivera, Benito Alén, Pablo Alonso-González, Yolanda González, \\ and Luisa González \\ Instituto de Microelectrónica de Madrid, CNM, CSIC, Isaac Newton 8, Tres Cantos, 28760 Madrid, Spain
}

(Received 27 February 2009; accepted 8 March 2009; published online 1 April 2009)

\begin{abstract}
We have developed a growth process that leads to the direct formation of self-assembled InAs quantum dots on $\operatorname{InP}(001)$ by solid-source molecular beam epitaxy avoiding the previous formation of quantum wires usually obtained by this technique. The process consists of a periodically alternated deposition of In and As correlated with InAs $(4 \times 2) \leftrightarrow(2 \times 4)$ surface reconstruction changes. Based on the results obtained by in situ characterization techniques, we propose that the quantum dots formation is possible due to the nucleation of In droplets over the $\operatorname{InAs}(4 \times 2)$ surface during the In deposition step and their subsequent crystallization under the As step. (C) 2009 American Institute of Physics. [DOI: 10.1063/1.3108087]
\end{abstract}

Semiconductor self-assembled quantum dots (QDs) have received much attention because of their application in advanced optical devices. ${ }^{1,2}$ In particular, the active elements based on the InAs/InP heteroepitaxial system are interesting for their use in laser devices emitting at $1.55 \mu \mathrm{m}$, compatible with optical fiber communications. ${ }^{3}$ The self-assembling of QDs is a strain-driven process that takes place in highly lattice-mismatched semiconductor materials. The strained material grows in a layer-by-layer mode up to a certain critical thickness and then the growth mode switches from two dimensional (2D) to three dimensional (3D) with the net result of elastic energy relaxation (Stranski-Krastanov process). The InAs/GaAs heteroepitaxial system is a well studied example of this kind of process. ${ }^{4}$ However, in the InAs/ $\operatorname{InP}(001)$ heteroepitaxial system growing under usual molecular beam epitaxy (MBE) conditions, quantum wires (QWRs) elongated along the [1 10$]$ direction are formed instead of QDs, which are in principle more efficient for elastic energy relaxation. Other authors have recently recognized the key role of the InAs As-rich $(2 \times 4)$ surface reconstruction in a growth model for QWR formation. ${ }^{5}$ Other works report the InAs/InP(001) QD formation using MBE but always through the evolution of previously formed QWRs: either by the ripening of the initial QWRs during substrate cooling down under arsenic overpressure ${ }^{6}$ or after substrate annealing under no arsenic flux at the $\operatorname{InAs}(4 \times 2)$ surface reconstruction. ${ }^{7}$ In a previous model we proposed that the growth of InAs on InP under a $(2 \times 4) \mathrm{V}$ element ended surface reconstruction produces a stress asymmetry at the InAs/InP interface, inducing an asymmetric relaxation process that finally results in QWR formation. ${ }^{8,9}$ According to our previous model, the built-in interface strain anisotropy could be inhibited if the surface would be terminated in III element. ${ }^{9}$ Therefore, if those conditions could be experimentally achieved, for example, growing InAs on the In-rich (4 $\times 2$ ) surface reconstruction, QDs instead of QWRs would be directly obtained in the $\operatorname{InAs} / \operatorname{InP}(001)$ system.

In this work we have developed a method to implement this idea. The method consists of depositing InAs in a pulsed mode under experimental conditions in which the surface shows most of the growth time a $(4 \times 2)$ surface reconstruc- tion. For that, an accurate control of the open-close time of the In and As cells and a high on/off ratio of the $\mathrm{As}_{4}$ beam equivalent pressure (BEP) in each pulse are essential. The samples were grown on InP (001) substrates in a homemade solid-source MBE system equipped with a specially designed As cell. This cell shows an on/off BEP $\left(\mathrm{As}_{4}\right)$ ratio $\geq 50$ with fast open-close response ( $\geq 0.1 \mathrm{~s})$ in each growth cycle. The QD formation process was studied during growth by in situ accumulated stress and reflection high energy electron diffraction (RHEED) measurements and their morphology and electronic properties were investigated by atomic force microscopy (AFM) and photoluminescence (PL).

The in situ accumulated stress $(\Sigma \sigma)$ measurements were made by monitoring during growth the curvature changes in thinned substrates $(\sim 190 \mu \mathrm{m})$ with a cantilever shape elongated either along the $[110]$ or $[1 \overline{1} 0]$ directions. The sample curvature was followed optically by the reflection of two parallel beams along the cantilever similar to the experiments reported in previous works. ${ }^{8-10}$ A large area digital camera was used for detection allowing the automated analysis of the beam deflections. The surface morphology was characterized by AFM in tapping mode with a Nanotec microscope. For PL characterization, the sample was optically pumped at $28 \mathrm{~K}$ with an $808 \mathrm{~nm}$ laser diode and the emitted light was dispersed with a $0.3 \mathrm{~m}$ focal length monochromator (Spectra Pro 2300i) and synchronously detected with an extended InGaAs photodiode (Hamamatsu G6122-03).

Previously to the InAs growth, an InP buffer layer of thickness above $150 \mathrm{~nm}$ was grown by MBE. Before opening the In cell, the $\mathrm{P}$ and As cell are switched producing an InAs wetting layer [thickness $\approx 1$ monolayer(ML)] due to As/P exchange on the surface at a substrate temperature $T_{S}$ $=515^{\circ} \mathrm{C} .{ }^{11}$ The InAs layer deposition was done at $T_{S}$ $=525^{\circ} \mathrm{C}$ through a pulsed mode where In and As cells are alternated. In each growth cycle, the In cell was opened during $1 \mathrm{~s}$ at an equivalent InAs growth rate of $0.1 \mathrm{ML} / \mathrm{s}$, and the As cell during $0.6 \mathrm{~s}$ at a $\operatorname{BEP}\left(\mathrm{As}_{4}\right)$ of $6.4 \times 10^{-6} \mathrm{mbar}$. During the $0.6 \mathrm{~s}$ that the As cell is open, the RHEED pattern shows a $(2 \times 4)$ surface reconstruction, whereas we observe a $(4 \times 2)$ surface reconstruction when the In cell is open. Therefore, the pulsed deposition is directly correlated with a 

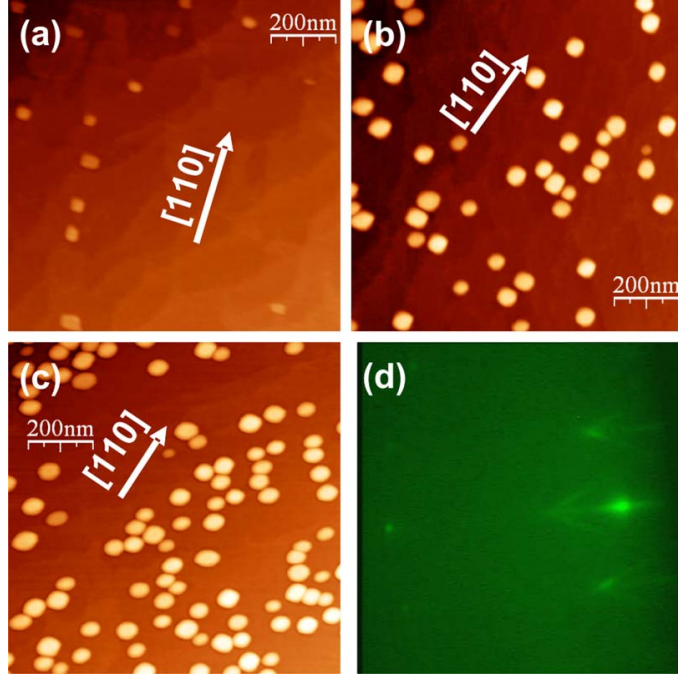

FIG. 1. (Color online) [(a)-(c)] $1 \times 1 \mu \mathrm{m}^{2} \mathrm{AFM}$ image (same $\mathrm{Z}$ scale) of InAs/InP (001) QDs for 1.5, 2.0, and 2.4 ML of InAs coverage, respectively; (d) 3D diffraction pattern corresponding to InAs/InP QDs at [1 $\overline{1} 0]$ electron incidence.

change between the $(4 \times 2)$ and $(2 \times 4)$ surface reconstructions. After the InAs layer was deposited, the sample was quickly cooled down under no As flux.

We produced samples with different amount of InAs deposition. Figures $1(\mathrm{a})-1(\mathrm{c})$ show $1 \times 1 \mu \mathrm{m}^{2}$ AFM images of samples corresponding to deposition of $0.5,1.0$, and 1.4 InAs ML, respectively. However, the actual amount of InAs exceeds that of the InAs deposited. The total amount of InAs in these samples is the result of the addition of the InAs deposited to the extra InAs formed at the interface by As/P exchange (around $1 \mathrm{ML}$ ). ${ }^{12}$ This yields an effective InAs coverage $\theta(\operatorname{InAs})$ of $1.5,2.0$, and $2.4 \mathrm{ML}$ for the samples shown in Figs. 1(a)-1(c), respectively.

The QD shape is a truncated pyramid with quasirectangular base. In the sample with 1.5 ML of InAs coverage, the density of QDs is $\rho=1.3 \times 10^{9} \mathrm{dots} / \mathrm{cm}^{2}$ and their side lengths along $\langle 110\rangle$ directions and height are $l_{1-10}$ $=40 \pm 5 \mathrm{~nm}, l_{110}=32 \pm 5 \mathrm{~nm}$, and $h=3.3 \pm 0.8 \mathrm{~nm}$, respectively. For the sample with $2.0 \mathrm{ML}$ of InAs coverage, $\rho$ $=5.0 \times 10^{9} \operatorname{dots} / \mathrm{cm}^{2}, \quad l_{1-10}=49 \pm 6 \mathrm{~nm}, \quad l_{110}=48 \pm 6 \mathrm{~nm}$, and $h=7.5 \pm 0.8 \mathrm{~nm}$. Finally, for the sample with $2.4 \mathrm{ML}$ of InAs coverage, $\rho=1 \times 10^{10} \mathrm{dots} / \mathrm{cm}^{2}, \quad l_{1-10}=51 \pm 4 \mathrm{~nm}$, $l_{110}=52 \pm 5 \mathrm{~nm}$, and $h=7.7 \pm 0.6 \mathrm{~nm}$. From these results we observe an increase in both size and density of the QDs with the InAs coverage.

We routinely observe [Fig. 1(d)] a 3D RHEED pattern corresponding to diffraction from QDs just after 0.5 ML (5 growth cycles) of InAs is deposited. Taking into account the wetting layer formed by As/P exchange, this corresponds to a $\theta$ (InAs) 1.5 ML. However, considering the InAs/InP lattice mismatch (3.2\%), we would not expect an InAs critical thickness for nanostructure formation as small as $1.5 \mathrm{ML}$, as we actually observe by RHEED. Notice that in the conventional growth procedure for QWR formation the asymmetrical relaxation process is detected for $\theta(\operatorname{InAs})=2.4 \mathrm{ML}$ as measured by in situ accumulated stress measurements. ${ }^{12}$

In order to get some understanding of the QD formation, we have performed in situ accumulated stress measurements during the growth of InAs by the above described process. Once the wetting layer was formed by As/P exchange, we

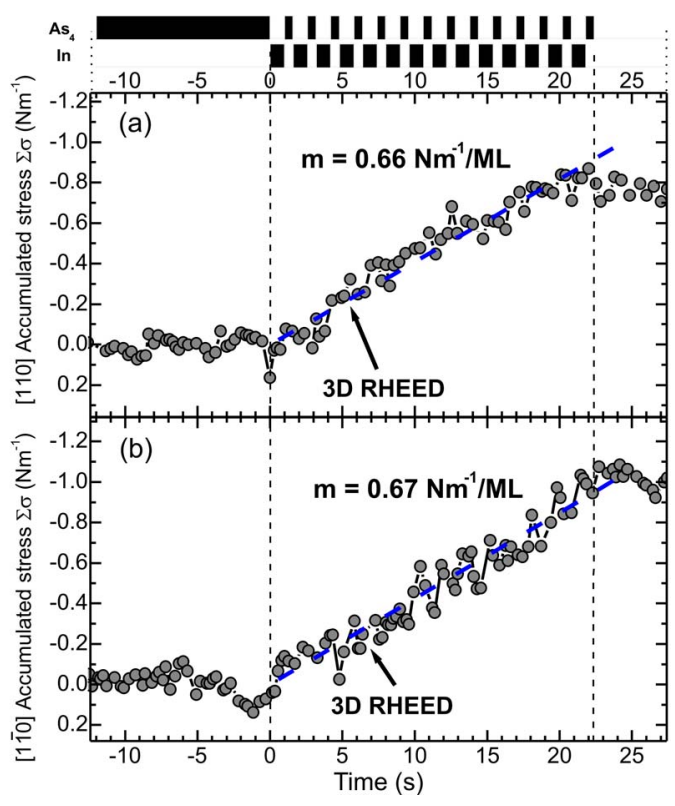

FIG. 2. (Color online) Accumulated stress measurements along (a) [110] and (b) $[1 \overline{1} 0]$ directions. The observation of the 3D RHEED pattern is indicated by arrows. The As and In cell state (on/off) is indicated on top.

deposited 1.4 ML of InAs, which takes $22.4 \mathrm{~s}$ due to the pulsed mode growth process. Figure 2 shows the change in

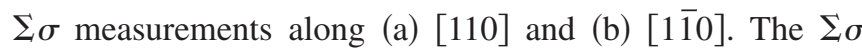
value corresponding to the InAs wetting layer at $T_{S}$ $=525{ }^{\circ} \mathrm{C}$ is taken as starting point $(\Sigma \sigma=0)$ in these measurements. The on/off sequence of the $\mathrm{As}_{4}$ and $\mathrm{In}$ cells is indicated at the top of Fig. 2. The arrows mark the onset of the corresponding 3D RHEED pattern. The variation rate of $\Sigma \sigma$ with InAs thickness $\left(\mathrm{N} \mathrm{m}^{-1} / \mathrm{ML}\right)$ obtained by a linear fit of the experimental data is also shown. The slope values correspond to $0.66 \pm 0.02 \mathrm{~N} \mathrm{~m}^{-1} / \mathrm{ML}$ and $0.67 \pm 0.02 \mathrm{~N} \mathrm{~m}^{-1} / \mathrm{ML}$ along [110] and [1 $\left.\overline{1} 0\right]$, respectively. Thus we observe a symmetric monotonous increase in the compressive stress along both $\langle 110\rangle$ directions as typical for QD formation. ${ }^{10}$ This result differs from the $\Sigma \sigma$ evolution during InAs growth for QWR formation in two aspects. First, we measured an average variation rate of $\Sigma \sigma$ with the InAs thickness during pseudomorphic growth of $0.77 \mathrm{~N} \mathrm{~m}^{-1} / \mathrm{ML}$ (close to the value of $0.78 \mathrm{~N} \mathrm{~m}^{-1} / \mathrm{ML}$ estimated using InAs and InP bulk elastic constants). ${ }^{8,9,13,14}$ Second, we observed that the QWRs relax stress only along [110] once $\theta$ (InAs) of $2.4 \mathrm{ML}$ is reached. The $\Sigma \sigma$ fluctuations observed during the pulsed InAs deposition are related to the surface stress contribution to $\Sigma \sigma$ produced by the periodical changes on the surface reconstruction $[(2 \times 4) \leftrightarrow(4 \times 2)]$ and must not be related to noise of the measurements. ${ }^{15}$

The variation rate of $\Sigma \sigma$ with deposited InAs and the early 3D RHEED pattern occurrence at $\theta(\operatorname{InAs})=1.5 \mathrm{ML}$ indicate that the nanostructure formation occurs from the beginning of InAs deposition in the pulsed growth mode followed here. At this stage, we propose that during In deposition on the In-rich $(4 \times 2)$ surface reconstruction, small In droplets are surfing over the InAs wetting layer (previously formed by As/P exchange). These droplets act as nucleation centers to capture the supplied As atoms and surrounding In atoms resulting into 3D InAs structures when the As cell is open. ${ }^{16,17}$ Therefore, InAs QDs would start forming from the onset of the InAs deposition as observed by RHEED. In fact, 


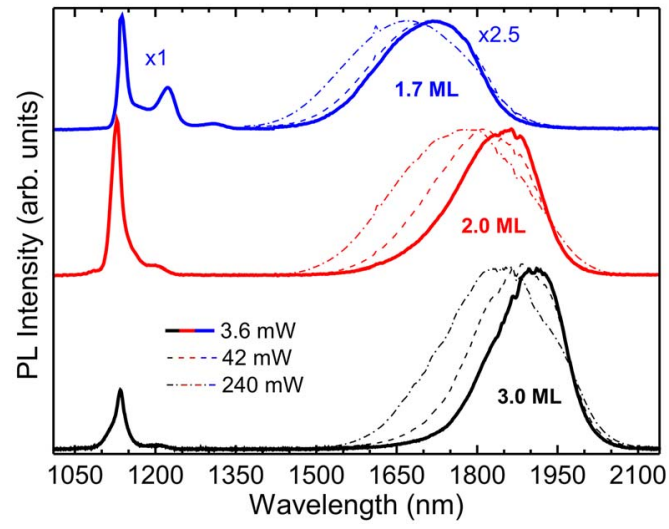

FIG. 3. (Color online) Normalized PL spectra of different InAs/InP(001) QD samples measured at $28 \mathrm{~K}$ under the indicated excitation powers.

both In incorporation in liquid droplets and further crystallization in 3D InAs nanostructures are processes that would result in a lower accumulated stress rate evolution in both $\langle 110\rangle$ directions compared with that expected for a InAs/InP pseudomorphic growth. Furthermore, this hypothesis would explain the size dispersion observed in the AFM images because while some InAs QDs are growing in size, more little In droplets are forming during the next growth cycles. On the other hand, our AFM results show that most of InAs QDs are formed at the upper side of surface steps as expected for self-assembled QDs. ${ }^{18}$ This result is not in contradiction with the random nucleation of small metallic droplets, ${ }^{19}$ which can migrate toward favorable sites during their crystallization into semiconductor nanostructures. ${ }^{20}$

Samples grown for QD formation were capped with a $30 \mathrm{~nm}$ thick InP layer for PL measurements. This layer was grown by atomic layer MBE (Ref. 21) after a quick sample cooling down to $T_{S}=380{ }^{\circ} \mathrm{C}$ under no As flux just after the QDs are formed. Figure 3 shows the $28 \mathrm{~K}$ PL spectra of three samples with different $\theta(\operatorname{InAs}): 1.7,2.0$, and $3.0 \mathrm{ML}$ for three different excitation powers: 3.6, 42, and $240 \mathrm{~mW}$. The high energy peaks are emitted from 1-3 ML thick quantum wells (or wetting layer), which we associate to the terraces between the QDs [see Figs. 1(a)-1(c)]. ${ }^{12,22}$ The quenching of the wetting layer emission when we increase the amount of InAs deposited could be due to the increase in size and density of the InAs QDs with the contribution from the thinning of the wetting layer similar to the InAs/InP QD growth by metal-organic vapor phase epitaxy. ${ }^{23}$ The emission from QDs shows a broadband (related to size dispersion) centered at lower energy: 1725,1850 , and $1900 \mathrm{~nm}$ for $\theta(\operatorname{InAs}) \sim 1.7$, 2.0, and 3.0 ML, respectively. These emission energies would correspond to previously calculated QD height range between 7 and $10 \mathrm{~nm}$ close to our AFM measurements. ${ }^{24}$ Despite the inhomogeneous broadening, as the excitation power increases, the low energy part of the PL band saturates and the high energy part increases as expected due to the excited state shell filling in QDs. ${ }^{25}$

In conclusion, we have obtained InAs QDs grown on InP (001) by solid source MBE using a growth process that does not involve the previous formation of QWRs. This is deci- sive for fabrication of single photon emitters and other optical devices working at optical telecommunication wavelengths. We found that the variation rate of $\Sigma \sigma$ with the amount of deposited InAs in the designed pulsed growth mode is lower than that expected for $2 \mathrm{D}$ growth. The early manifestation of the 3D RHEED pattern suggests that the QD formation occurs from the onset of InAs deposition once the wetting layer is formed by As/P exchange. We propose that the QDs are the result of the crystallization under the As flux cycles of In droplets formed during In deposition cycles throughout the pulsed InAs growth process. In addition, the QD size dispersion evidenced by AFM and PL measurements supports our hypothesis.

The authors acknowledge the financial support of the Spanish MICINN (TEC2008-06756-C03-01), ConsoliderQOIT (CSD2006-0019), and CAM (S-505/ESP/000200). P.A.G. and A.R. thank the I3P program. B.A. thanks the Ramón y Cajal program.

${ }^{1}$ A. Badolato, K. Hennessy, M. Atatüre, J. Dreiser, E. Hu, P. M. Petroff, and A. Imamouglu, Science 308, 1158 (2005).

${ }^{2}$ R. M. Stevenson, R. J. Young, P. Atkinson, K. Cooper, D. A. Ritchie, and A. J. Shields, Nature (London) 439, 179 (2006).

${ }^{3}$ F. Suárez, D. Fuster, L. González, Y. González, J. M. García, and M. L. Dotor, Appl. Phys. Lett. 89, 091123 (2006).

${ }^{4}$ D. Leonard, K. Pond, and P. M. Petroff, Phys. Rev. B 50, 11687 (1994).

${ }^{5}$ T. J. Krzyzewski and T. S. Jones, Phys. Rev. B 78, 155307 (2008).

${ }^{6}$ E. Dupuy, P. Regreny, Y. Robach, M. Gendry, N. Chauvin, E. Tranvouez, G. Bremond, C. Bru-Chevallier, and G. Patriarche, Appl. Phys. Lett. 89, 123112 (2006).

${ }^{7}$ H. Yang, P. Ballet, and G. J. Salamo, J. Appl. Phys. 89, 7871 (2001).

${ }^{8}$ J. M. García, L. González, M. U. González, J. P. Silveira, Y. González, and F. Briones, J. Cryst. Growth 227, 975 (2001).

${ }^{9}$ M. U. González, L. González, J. M. García, Y. González, J. P. Silveira, and F. Briones, Microelectron. J. 35, 13 (2004).

${ }^{10}$ J. M. García, J. P. Silveira, and F. Briones, Appl. Phys. Lett. 77, 409 (2000).

${ }^{11}$ M. U. González, J. M. García, L. González, J. P. Silveira, Y. González, J. D. Gómez, and F. Briones, Appl. Surf. Sci. 188, 188 (2002).

${ }^{12}$ D. Fuster, B. Alén, L. González, Y. González, J. Martínez-Pastor, M. U. González, and J. M. García, Nanotechnology 18, 035604 (2007).

${ }^{13}$ D. Fuster, M. U. González, L. González, Y. González, T. Ben, A. Ponce, and S. I. Molina, Appl. Phys. Lett. 84, 4723 (2004).

${ }^{14}$ J. M. Ulloa, P. M. Koenraad, D. Fuster, L. González, Y. González, and M U. González, Nanotechnology 19, 445601 (2008).

${ }^{15}$ D. Fuster, M. U. González, Y. González, and L. González, Surf. Sci. 600, 23 (2006).

${ }^{16}$ J. S. Kim and N. Koguchi, Appl. Phys. Lett. 85, 5893 (2004).

${ }^{17}$ J. H. Lee, Zh. M. Wang, and G. J. Salamo, J. Phys.: Condens. Matter 19, 176223 (2007).

${ }^{18}$ S. O. Cho, Zh. M. Wang, and G. J. Salamo, Appl. Phys. Lett. 86, 113106 (2005).

${ }^{19}$ Z. Y. AbuWaar, Zh. M. Wang, J. H. Lee, and G. J. Salamo, Nanotechnology 17, 4037 (2006).

${ }^{20}$ P. Alonso-González, L. González, D. Fuster, Y. González, A. G. Taboada and J. M. Ripalda, Cryst. Growth Des. 9, 1216 (2009).

${ }^{21}$ F. Briones, L. González, and A. Ruiz, Appl. Phys. A: Solids Surf. 49, 729 (1989).

${ }^{22}$ B. Alén, D. Fuster, Y. González, L. González, and J. Martínez-Pastor, Appl. Phys. Lett. 89, 233126 (2006).

${ }^{23}$ B. Bansal, M. R. Gokhale, A. Bhattacharya, and B. M. Arora, J. Appl. Phys. 101, 094303 (2007).

${ }^{24}$ M. Holm, M.-E. Pistol, and C. Pryor, J. Appl. Phys. 92, 932 (2002).

${ }^{25}$ P. Hawrylak, G. A. Narvaez, M. Bayer, and A. Forchel, Phys. Rev. Lett. $\mathbf{8 5}, 389$ (2000). 